\title{
Über die Löslichkeit der Salze isotoper Elemente.
}

\author{
Von K. FaJans and J. Fischler.
}

\section{Einleitung. ${ }^{1}$}

Durch die Beobachtungen von H. Mc Cor und W. Ross 2 , B. Boltwood ${ }^{3}$, W. Marckwald ${ }^{4}$, B. Kentman ${ }^{5}$, F. Soddy ${ }^{6}$ u. A. wurde zuerst gezeigt, daB es nicht gelingt, gewisse Radioelemente von manchen gewöhnlichen Elementen aut chemischem Wege zu trennen. ${ }^{7}$ D. Strömнour und The SVEDBFRG ${ }^{8}$, sowie Soddy ${ }^{9}$ haben die ersten theoretischen Ansichten über diese Frscheinung geäuBert, und sie diente als eine der Grundlagen der am Anfange des Jahres 1913 von FAJANs ${ }^{10}$ und SodDr ${ }^{11}$ aufgestellten allgemeinen Theorie der chemischen Eigenschaften aller Radioelemente und ihres Verhältnisses zum periorliscken System. ${ }^{\mathbf{1 2}}$ Nach dieser Theorie gibt es nämlich sogenannte isotope Elemente, die bei Atomgewichtsunterschieden bis zu 8 Einheiten und verschiedenen radioaktiven Eigenschaften so weitgehend gleiche chemische Eigenschaften besitzen, daß ein Gemisch solcher Elemente chemisch als

1 Dieses und das nächste Kapitel beziehen sich auch auf die folgende Arbeit von FAJANS und LEMBERT.

2 Journ. Amer. Chem. Soc. 29 (1907), 1709.

${ }^{3}$ Amer. Journ. Science 95 (1908), 365.

4 Ber. deutsch. Chem. Ges. 41 (1908), $49 ; 43$ (1910), 3420.

5 Jahrb. d. Radioakt. u. Elektronik 6 (1909), 265.

6 Journ. Chem. Soc. 99 (1911), 72.

`Näheres über die Entwicklung dieses Forschungsgebietes vgl. den zusammenfassenden Bericht von K. FAJANs, Physikal.Zeitschr. 16 (1915), 456. Im folgenden bezeichnet mit $\mathbf{F}$.

s Z. anorg. Chem. 61 (1909), 338; 63 (1909), 197.

1.c.

10 Physik. Zeitschr. 14 (1913), 136 ; Ber. deutsch. Chem. Ges. 46 (1913), 422.

11 Chem. News 107 (1913), 97; Jahrb. d. Radioakt. u. Elektronik 10 (1913), 188.

1* Vgl. auch A. S. Russelu, Chem. News 107 (1913), 49. 
ein Element betrachtet werden darf, dem nur eine Stelle im periodischen System zukommt. Die Möglichkeit der Anwendung dieser zunächst nur durch das Verhalten der Radioelemente gestützten ${ }^{1}$ Theorie auch auf gewöhnliche Elemente wurde von Fajans und SodDy diskutiert. In der Tat konnte bald darauf, in Bestätigung einer der Folgerungen der Theorie, durch direkte Atomgewichtsbestimmungen gezeigt werden, da $\beta$ das aus radioaktiven Mineralien abgeschiedene Blei ein anderes Atomgewicht als das gewöhnliche Blei besitzt, obwohl es weder in qualitativ-chemiseher noch in spektroskopischer Hinsicht vom gewöhnlichen Blei zu unterscheiden ist. Th. W. Richards und M. Lembert ${ }^{2}$, O. Hönigschmid and Frl. Sт. Horovitz: ${ }^{3}$ M. CurIE ${ }^{4}$ haben für das Atomgewicht des aus verschiedenen Uranmineralien abgeschiedenen Bleies Werte zwischen 206.0 und 206.9 erhalten ${ }^{5}$, während das Atomgewicht des gewöhnlichen Bleies $(\mathrm{Pb}) 207.20$ beträgt. $^{6}$

Diese Beobachtungen werden dadurch erklärt ", daß in den Uranmineralien das jedenfalls sehr stabile Umwandlungsprodukt des Radium F - das Radium G - mit dem Atomgewicht etwa 206,0 im Laufe geologischer Zeiten durch die Zersetzung des Urans akkumuliert wird, und da seine chemischen Eigenschaften nach der Theorie denen des Bleies außerordentlich ähnlich sind - Radium $G^{8}$ ist ein Glied der Bleiplejade oder isotop mit Blei -, so wird es zusammen mit gewöhnlichem Blei aus diesen Mineralien abge-schieden. Je nach dem Mengenverhältnis des Radium $\mathrm{G}$ und $\mathrm{Pb}$ kann deshalb das Gemisch Atomgewichte aufweisen, die zwischen 206.0 und 207.2 liegen.

1 A. Funck., Journ. Chem. Soc. 103 (1913), 381 u. 1052. - W. Metzener, Ber. deutsch. Chem. Ges. 46 (1913), 979. - G. v. Hevesy u. F. Pangth, Wiener Ber. 122 (1913), IIa, 993. - P. BEER, Dissertation. Karlsruhe 1914.- O. GöHRING, Dissertation. Karlsruhe 1914.

2 Z. anorg. Chem. 88 (1914), 429.

3 Wiener Ber. 123 (1914), IIb, 1033; 123 (1914), IIa, 2407.

4 Comp. rend. 158 (1914), 1676.

${ }^{5}$ Auf der anderen Seite gibt es Anzeichen dafür, daß Blei aus Thormineralien ein höheres Atomgewicht als das gewöhnliche Blei besitzt. F. SoDDY u. H. HyMarN, Journ. Chem. Soc. 105 (1914), 1402. - F. SoDDY, Nature 94 (1915), 615; vgl. auch die Diskussion dieser Resultate F., S. 474.

- G. P. Baxter und L. Grover, Journ. Amer. Chem. Soc. 37 (1915), 1027.

7 Eine vollständige Diskussion vgl. F. l. e.

s Radium G (Radiumblei) bildet den Hauptbestandteil des sogenannten Uranbleies, dessen zweiter Bestandteil das Aktiniumblei ist. 
Die Entdeckung von Bleiarten ${ }^{1}$ mit verschiedenem Atomgewicht stellt die experimentelle Forschung vor ganz neue Aufgaben. Während in den meisten bekannten Fällen die isotopen Elemente durch ihre verschiedenen radioaktiven Eigenschaften sehr leicht roneinander und nebeneinander zu unterscheiden sind, scheint das beim RaG und $\mathrm{Pb}$ nicht möglich zu sein, da sich bei keinem von ihnen bis jetzt radioaktive Eigenschatten nachweisen ließen. ${ }^{2}$ Die Atomgewichtsbestimmung und andere die Unterschiede des Atomgewichtes benutzenden Methoden werden deshalb den einzigen Weg bilden, um in diesem und ähnlichen Fällen die isotopen Elemente zu erkennen und zu unterscheiden. Da die direkte Atomgewichtsbestimmung meistens recht zeitraubend und schwierig ist, wird es von großer Bedeutung sein, zu diesem Zweck andere einfachere Methoden auszuarbeiten, und eine gleich zu erwähnende einfache Überlegung, die in dieser und in der folgenden Arbeit von Fajans and Lembert bestätigt wird, zeigte, daß sich eine sehr geeignete Methode auf die Löslichkeit des Salze isotoper Elemente gründen läßt.

Außer diesem praktischen Gesichtspunkt hat noch folgender theoretischer zu diesen Arbeiten Veranlassung gegeben. In der Soddr-Fajansschen Theorie wurde zuerst angenommen, daß die Isotopen qualitativ-chemisch nicht unterscheidbar sind. Dies ist sicherlich mit weitgehender Genauigkeit richtig und man wird die qualitativ-chemischen Eigenschaften der Isotopen wohl immer als praktisch gleich betrachten dürfen. Eine nähere theoretische Analyse ${ }^{3}$ der Eigenschaften der Isotopen auf Grund des RtTHeRForD-BoHrschen Atommodells ${ }^{4}$ und der Theorie fester Körper führte jedoch zu dem Resultat, daß zwischen allen Eigenschaften der Isotopen mit Ausnahme der Kernladung doch gewisse, wenn auch meistens sehr kleine Unterschiede anzunehmen sind. ${ }^{\mathbf{5}}$ Der experimentellen Forschung

1 Von den zwei Ausdrücken Bleiarten und Bleisorten (FAJANS, Ber. deutsch. Chem. Ges. 46 (1913), 430 werden wir von jetzt ab nur den ersten' benutzen. Vgl. Kapitel IX der folgenden Arbeit.

2 Die Radioaktivität des aus Uranmineralien abgeschiedenen Bleies stammt von den kurzlebigen Umwandlungsprodukten des ebenfalls zur Bleiplejade gehörenden Radium D.

${ }^{3}$ K. Fajaks, Elster-Geitel-Festschrift (1915), 623.

4 Sir E. Rutherford, Phil. Mag. 27 (1914), 488 - N. Bohr, Phil. Mag. 26 (1913), 1 u. 476; 27 (1914), 506.

5 Vil. auch F. A. Lindemann, Nature 95 (1915), 7. 
erwächst deshalb die wichtige Aufgabe, die Eigenschaften der Isotopen mit möglichst großer Genauigkeit zu vergleichen, um den Grad ihrer Übereinstimmung zu untersuchen und eventuell die zu erwartenden Unterschiede nachzuweisen. Die Löslichkeit der Salze gehört nun zu denjenigen Figenschaften, in welchen ähnliche Elemente, wie z. B. die Elemente der seltenen Erden, sonst besonders große Unterschiede zeigen, und es schien deshalb wünschenswert, die isotojen Bleiarten in dieser Richtung näher zu untersuchen.

Schließlich bietet das Verhalten der Gemische ron Isotopen beim Lösungsvorgange sowohl in theoretischer wie auch in praktischer Hinsicht gewisses Interesse, und auch mit dieser Frage stehen die ausgeführten Versuche im Zusammenhang.

\section{Plan der Untersuchung.}

Der Plan dieser und der folgenden Untersuchung stützté sich auf folgende Überlegung. ${ }^{1}$ Unter der Voraussietzung, daß gesättigte Lösungen einander entsprechender Salze isotoper Elemente die gleiche molare Zusammensetzung haben und daß sie in gleichen Volumina die gleiche Anzahl Mole der Salze enthalten, folgt aus dem verschiedenen Molekulargewicht der Salze, daß sowohl ihre in Grammen pro Liter ausgedrückte Löslichkeit als auch die Dichte der gesättigten Lösungen verschieden sein müssen. Das Ziel der Untersuchung war nun die Feststellung und Messung dieses Unterschiedes der Dichte, wodurch sowohl die obigen Voraussetzungen geprüft, wie auch eine Methode zur relativen Atomgewichtsbestimmung der Isotopen ausgearbeitet werden sollte.

Von allen Bleisalzen ist das Nitrat für diese Untersuchung das am meisten geeignete, weil es sowohl eine relativ hohe Löslichkeit im Wasser besitzt, als auch verhältnismäßig leicht in reinem Zustande herzustellen ist. Aus folgender Rechnung war zu ersehen, mit welcher Genauigkeit man die Versuche ausführen muß, um der gestellten Aufgabe gerecht zu werden.

Wir benutzten zu diesen Versuchen zwei Bleiarten, nämlich ein Bleipräparat aus Carnotit (Colorado), dessen Atomgewicht

1 Fa.Tans, Zeitschr. f. Elektrochem. 20 (1914), 459. 
Th. W. Richards und M. Lembert zu 206.59 bestimmt haben ${ }^{1}$ und gewöhnliches Blei des Handels, für dessen Atomgewicht dieselben Autoren in Parallelversuchen den Wert 207.15 fanden. Der Unterschied im Atomgewicht beträgt somit 2.7\%. Nun enthält die gesättigte Lösung des Bleinitrats bei $25^{0}$ pro $10 \mathrm{~cm}$ etwa $5 \mathrm{~g}$ $\mathrm{Salz}^{2}$, also etwa $3 \mathrm{~g}$ Metall. Zwischen je $10 \mathrm{ccm}$ der gesăttigten Lösungen der Nitrate der benutzten Bleiarten sollte also auf Grund obiger Annahmen ein Unterschied um etwa $\frac{2.7 \cdot 3}{1000}=0.008 \mathrm{~g}$ bestehen, der sich natürlich in einem Pyknometer mit Hilfe einer guten Wage mit genügender Genauigkeit leicht bestimmen läßt. Für die exforderliche Konstanz der Temperatur während der Finstellung des Lösungsgleichgewichts ergibt sich iolgendes: Die Löslichkeit des Bleinitrats ändert $\operatorname{sich}^{3}$ um etwa $1 \%$ pro $1^{0}$; um also die wünschenswerte Genauigkeit von $1 / 10 \%$ zu erreichen, muß die Temperatur innerhalb $1 / 100$ konstant gehalten werden, was sich auch erreichen läßt. Das Hauptaugenmerk mußte somit auf die Herstellung reiner reproduzierbarer gesättigter Salzlösungen gerichtet werden. Es war dabei einerseits darauf zu achten, daß die Löslichkeit unterhalb einer gewissen Größe des Kristallkornes durch diese beeinflußt wird und daß andererseits jede Verunreinigung die Resultate stark beeinflussen kann, indem sie selbst mitgelöst wird und sowohl das Gewicht der Lösung als auch die Löslichkeit des Bleinitrates ändern kann.

Es lag in dem Plan unserer Untersuchung, zunächst in Vorversuchen ohne Anwendung der größtmöglichen VorsichtsmaßBregeln bei der Herstellung der Präparate und bei den Messungen zu prüfen, ob der gesuchte Dichteunterschied überhaupt auftritt, und dann erst an seine genaue Bestimmung zu gehen. Unsere Anfang Mai 1914 begonnenen Versuche mußten aber bei Ausbruch des Krieges abgebrochen werden und es ist uns nur gelungen, den ersten Teil des Planes zur Ausführung zu bringen, während der

1. Es ist dasselbe Präparat, das mir von Herrn Professor F. GIEsec in größeren Quantitäten freundlichst überlassen wurde und von dem ich einen Teil den Herren Richards und LEMBERT zur Atomgewichtsbestimmung übergeben habe. Über die Herstellung des Präparates vgl. Fajass, Heidelberger Akademie, Abhandlung 11 A (1914). K. F.

2 A. C. Cummina, Zeitschr. f. Elektrochem. 13 (1907), 19. - W. K. Levis, Dissertation, Breslau 1908, S. 16.

${ }^{3}$ ABEGG, Handbuch der anorgan. Chemie III, 2, S. 711. 
zweite Teil der folgenden Untersuchung des einen von uns mit M. Lembert vorbehalten bleiben mußte. Wegen des nur orientierenden Charakters der Arbeit sollen unsere Versuche nur kurz und auszugsweise beschrieben werden, wobei wir besonders diejenigen Punkte berücksichtigen werden, die für die Hauptversuche der folgenden Untersuchung von Nutzen gewesen sind.

\section{Versuchsanordnung.}

Die Einstellung des Lösungsgleichgewichtes erfolgte in einem viereckigen Thermostat von etwa 60 Liter Inhalt, der mit Gasheizung und einem gewöhnlichen OsTwaLdschen Toluolregulator versehen war. Die Schwankungen um die mittlere Temperatur $24.43^{\circ}$ betrugen $\pm 0.02^{\circ}$. In der Mitte des Thermostaten rotierte eine horizontale Welle, an die senkrecht zu ihr Rührflügel und sechs zylindrische Behälter für die die Lösung enthaltenden Glasrohre angelötet waren. Die zu untersuchenden Salze mit dem als Lösungsmittel dienenden Wasser befanden sich in Reagenzröhren aus Jenaer Glas (innerer Durchmesser $21 \mathrm{~mm}$, äußerer $24 \mathrm{~mm}$, Länge $13 \mathrm{~cm}$, Volumen etwa $40 \mathrm{~cm}$ ), die mit Gummistopfen verschlossen wurden. Nach der Einstellung des Gleichgewichtes wurden die Röhren aus ihren Behältern herausgenommen und im Thermostaten aufgehängt. Wenn der Gummistopfen nicht durch eine Gummikappe vor Berührung mit dem Wasser des Thermostaten geschützt worden war, wurde er mit destilliertem Wasser außen abgespült und mit Seidenpapier abgetrocknet. Nach dem Absetzen des Bodenkörpers wurde die klare Lösung in ein gewöhnliches SPRENGEL-Ostwaldsches Pyknometer von etwa $10 \mathrm{ccm}$ Inhalt durch Vermittlung eines mit Gummischlauch an die Pyknometerspitze betestigten Glasröhrchens (Glas an Glas) etwas über die Marke angesaugt. Der Gummischlauch mit dem Röhrchen wurde dann abgenommen und die Einstellung auf die Marke ${ }^{1}$ durch Berühren der Pyknometerspitze mit Fließpapier erreicht. Das Pyknometer befand sich vor der Füllung in einem Luftbad im Thermostaten und wurde nach der Füllung sofort gewogen. Die Wägungen wurden auf einer gewöhnlichen analytischen Wage mit der für unsere Versuche genügenden Genauigkeit von

1 Zur Erreichung schärferer Einstellung befand sich die Marke in einem kapillar verengten Teil des Pyknometerrohres (wie in Fig. 3 der folgenden Abhandlung).

Z. anorg. u. allg. Chem. Bd. 95. 
$0.1 \mathrm{mg}$ ausgeführt. Nach dem Versuch wurde das Pyknometer entleert, gründlich mit Wasser gewaschen und durch Erhitzen unter Durchsaugen trockener Luft getrocknet.

\section{Präparate.}

Ausgenommen einige Löslichkeitsbestimmungen an Chloriden wurden alle Versuche mit den Nitraten der Bleie ausgeführt. Als Ausgangsmaterial für die Präparate des Carnotitbleies diente uns ein ziemlich stark verunreinigtes Chlorid. ${ }^{1}$ Beim gewöhnlichen Blei gingen wir entweder von reinem Bleichlorid oder Nitrat (bezogen von Merck) aus. Der Bequemlichkeit halber wollen wir das Blei aus Carnotit mit $\mathrm{Pb}^{\prime}$, das gewöhnliche Blei wie immer mit $\mathrm{Pb}$ bezeichnen. ${ }^{2}$ Sowohl das $\mathrm{Pb}^{\prime} \mathrm{Cl}_{2}$ als auch das $\mathrm{PbCl}_{2}$ wurde folgendem Reinigungsprozeß unterworfen. Das Chlorid wurde in Wasser aufgeschlemmt und durch Einleiten von $\mathrm{H}_{2} \mathrm{~S}$ in einem verschlossenen Rundkolben mit Zu- und Ableitungsrohr in Sulfid verwandelt. Dieses wurde abfiltriert, gewaschen, in $\mathrm{HNO}_{3}$ gelöst. Die von etwas Schwefel und Sulfat abfiltrierte Lösung wurde mit $\mathrm{H}_{2} \mathrm{SO}_{4}$ gefällt, das abfiltrierte und ausgewaschene Sulfat in ammoniakalischem Ammonacetat gelöst, mit $\mathrm{H}_{2} \mathrm{~S}$ gefällt und das Sulfid wieder in $\mathrm{HNO}_{3}$ gelöst. Die filtrierte Lösung wurde auf dem Wasserbade zur Trockne abgedampft, das Nitrat mit Wasser aufgenommen, von kleinen Mengen des in Lösung gewesenen Sulfats abfiltriert und dann verschieden verarbeitet, je nachdem das Chlorid oder Nitrat gewonnen werden sollte. Für die Verarbeitung auf Chlorid wurde die Nitratlösung mit konzentriertem $\mathrm{HCl}$ in der Kälte gefällt, das Chlorid abgesaugt, mit eiskaltem Wasser gewaschen und dann dreimal aus Wasser kristallisiert. Diese Chloridpräparate, die zu verhältnismäßig rohen Versuchen gedient haben, machen keinen Anspruch auf besondere Reinheit.

Um reines Nitrat zu gewinnen, wurde die oben erwähnte Nitratlösung zur Befreiung von feinen Filterpapierteilchen durch einen Platin-Gooch-Neubaner-Tiegel ${ }^{3}$ filtriert und dann aus Wasser kristal. lisiert: zunächst noch einmal in Gefäßen aus Jenaglas und dann

1 Vgl. FaJaNs, Heidelberger Akademie 1914, A. 11, S. 17.

2 Näheres über Nomenklaturvorschläge für Isotopen vgl. Kapitel IX der folgenden Abhandlung.

${ }^{3}$ A. Stähler, Handb. d. Arbeitsmethoden i. d. anorg. Chem. Bd. 1, S. 665. 
mehrmals in Platinschalen, wobei die Kristalle von der ihnen anhaftenden Mutterlauge durch Zentrifugieren in einem Platin-GoochTiegel ${ }^{1}$ befreit wurden. Die Messungen mit den ersten Präparaten, die noch große Schwankungen zeigten, wollen wir übergehen und werden nur die an unseren letzten vier Präparaten ausgeführten Bestimmungen beschreiben.

A. $\mathrm{Pb}^{\prime}\left(\mathrm{NO}_{3}\right)_{2}$, erhalten auf dem oben beschriebenen Wege nach sechsmaligem Kristallisieren in Platin.

B. Präparat A noch zweimal kristallisiert.

C. $\mathrm{Pb}\left(\mathrm{NO}_{i j}\right)_{2}$, erhalten wie $\mathrm{A}$ aus dem Chlorid.

D. $\mathrm{Pb}\left(\mathrm{NO}_{3}\right)_{2}$, gewonnen durch viermaliges Kristallisieren des käuflichen Nitrats in Platingefäßen.

Es sei noch erwähnt, daß alle bei den obigen Operationen gebrauchten Reagenzien die Merckschen ,pro analysi“" waren. Das für die Kristallisationen und Füllung der Versuchsrohre benutzte Wasser wurde durch nochmalige Destillation des destillierten Wassers des Instituts unter Benutzung eines zu diesem Zweck schon lange im Gebrauch stehenden Kühlers aus Jenaer Glas gewonnen. Verwendet wurde nur das mittlere Destillat.

\section{Löslichkeit der Chloride der Bleie.}

Bevor wir an die subtileren Versuche mit den Nitraten gingen, wollten wir noch im Falle der Chloride feststellen, ob nicht zwischen der molaren Löslichkeit der Salze der Isotopen entgegen allen Er. wartungen leicht feststellbare Unterschiede auftreten: war es doch das erste Mal, daß die chemischen Eigenschaften der Isotopen durch direkte Messungen verglichen werden konnten. Die im vorigen Kapitel erwähnten Chloridpräparate wurden dazu mit Wasser geschüttelt. Es wurden mit $\mathrm{Pb}^{\prime} \mathrm{Cl}_{2}$ und $\mathrm{PbCl}_{2}$ je zwei Versuche gemacht; bei dem einen wurde die Mischung bis etwa $80^{\circ}$ erhitzt, und dann das Gleichgewicht durch Abkühlen im Thermostateri $\left(25^{\circ}\right)^{2}$ erreicht, bei dem zweiten wurde das Salz mit Wasser von Zimmertemperatur übergossen, und das Gleichgewicht von der anderen Seite eingestellt. ${ }^{3}$ Nach dreitägigem Schütteln wurde der Bodenkörper

1 1. c. S. 705 .

2 Der bei diesen Versuchen benutzte Toluolregulator erlaubte die Temperatur nur bis auf $\pm 0.1^{\circ}$ konstant zu halten.

3 Hier und im folgenden werden die zwei Arten der Einstellung des Gleichgewichtes durch ,heiB“" und ,kalt" bezeichnet. 
absitzen gelassen, dann mit derselben Pipette je $20 \mathrm{ccm}$ der Lösung abpipettiert und der Bleigehalt in der üblichen Weise ${ }^{1}$ durch Fällung mit Schwefelsäure und Filtrieren durch einen Porzellan-AsbestGooch-Tiegel bestimmt.

Das Gewicht des Sulfats war:

$$
\begin{array}{ccc} 
& \text { Carnotitblei } & \text { Gewöhnliches Blei } \\
\text { „heiB“ . . } 0.2368 \mathrm{~g} & 0.2367 \mathrm{~g}^{2} \\
\text { „,kalt" . . } 0.2366 \mathrm{~g} & 0.2370 \mathrm{~g}
\end{array}
$$

Wie die U̇bereinstimmung zwischen den Versuchen „heiß“ und „kalt" zeigt, ist das Gleichgewicht erreicht worden, und es ist auch kein sicherer Unterschied zwischen den beiden Bleien feststellbar. Theoretisch müßte im Falle der gleichen molaren Löslichkeit das Resultat beim gewöhnlichen Blei um $0.44 \mathrm{mg}$ höher sein, als bei dem anderen, was aber bei der unbeträchtlichen Genauigkeit dieser Versuche sich kaum bemerkbar machen konnte. Man kann also aus diesen Versuchen folgern, daß die molare Löslichkeit der Chloride der untersuchten zwei Bleiarten innerhalb der Versuchsfehler von etwa $1 / 2 \%$ gleich ist. ${ }^{3}$

\section{Löslichkeit der Nitrate der Bleie.}

Die Resultate unserer meisten Versuche mit den Nitraten sollen durch direkte Angabe des Gewichtes desselben mit der betreffenden gesättigten Lösung gefüllten Pyknometers wiedergegeben werden. Durch vielmalige Wägungen im Laufe der Versuche überzeugten wir uns, daß das Gewicht des leeren Pyknometers innerhalb von $0.5 \mathrm{mg}$ konstant blieb und daß auch sein Volumen sich nicht änderte, denn die Wägungen des mit Wasser gefüllten Pyknometers differierten im äußersten Falle um $1 \mathrm{mg}$. Die Berücksichtigung des je nach Druck und Temperatur etwas verschiedenen Luftauftriebes war bei der von uns erstrebten Genauigkeit überflüssig.

1 F. P. Treadwell, Quantitative Analyse.

2 Entsprechend $10.9 \mathrm{~g} \mathrm{PbCl}_{2}$ im Liter Lösung, was mit dem Resultat (10.8 g) anderer Autoren übereinstimmt. Vgl. ABEGG, Handb. d. anorg. Chemie III, 2, S. 649 .

${ }^{8}$ Dieses Resultat ist schon im Mai 1914 mitgeteilt worden (FAJANs, Zeitschr. t. Elektrochem. 20 (1914), 459. 
Die zur Einstellung des Gleichgewichtes nötige Zeit ergibt sich aus folgenden Versuchen. Zwei Rohre wurden mit demselben Nitratpräparat und mit einer zu seiner vollständigen Lösung bei $24.4^{\circ}$ ungenügenden Wassermenge beschickt, und das eine (,kalt") direkt in den Thermostaten, während im anderen (,heiß") durch Erhitzen das ganze Salz gelöst, und dann das Rohr in den Thermostaten eingeführt wurde.

Nach etwa 15stündigem Rühren wog dasselbe mit den zwei Lösungen gefüllte Pyknometer

$$
\begin{aligned}
& \text {,heiß“. . . . . } 21.7006 \mathrm{~g} \\
& \text {,kalt“ . . . . . } 21.6914 \mathrm{~g} \text {. }
\end{aligned}
$$

Ein anderes Pyknometer:

$$
\begin{array}{llll}
\text {,heiß“. . . . . . . . } 22,7556 \mathrm{~g} \\
\text {,kalt" . . . . . . . } 22.7476 \mathrm{~g} \text {. }
\end{array}
$$

Bei einem anderen Versuch erhielt man nach zweitägigem Rühren in dem zweiten der erwähnten Pyknometer:

$$
\begin{aligned}
& \text { „heiß" . . 22.7494 g „ „kalt" . . } 22.7488 \mathrm{~g}
\end{aligned}
$$

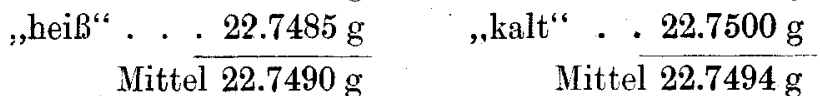

Nach 15 Stunden ist die heiße Lösung noch beträchtlich schwerer als die kalte, nach 2 Tagen ist kein die Versuchsfehler überschreitender Unterschied zwischen beiden festzustellen. Das Gleichgewicht ist also erreicht. Es wurde deshalb bei allen Versuchen mindestens 2 Tage gerührt. Die letzteren Versuche geben auch die bei demselben Präparat und verschiedenen Füllungen erreichte Genauigkeit an. Diese orientierenden Versuche wurden noch nicht mit unseren reinsten Präparaten ausgeführt. Jetzt seien die mit diesen erhaltenen Resultate erwähnt.

Zunächst sollen die Resultate der direkten gewichtsanalytischen Bestimmung des Bleigehaltes der gesättigten Lösungen der Präparate $\mathrm{A}$ und $\mathrm{C}$ angegeben werden. Mit derselben Pipette, deren Inhalt zu $3.0445 \mathrm{ccm}$ bestimmt worden war, wurden die Lösungen entnommen und auf die bei den Chloridversuchen angegebene Weise nach der Sulfatmethode analysiert. Der Bleisulfatniederschlag wog beim

$$
\begin{aligned}
& \text { gewöhnlichen Blei (C) . } 1.4740 \mathrm{~g} \\
& \text { Carnotitblei (A) . . . } 1.4693 \mathrm{~g} \text {. }
\end{aligned}
$$


Dividiert man diese Resultate durch die Molekulargewichte der Sulfate der benutzten Bleiarten, so erhält man

$$
\text { für } \mathrm{C} \frac{1.4740}{303.21}=0.004861 \text {; für } \mathrm{A} \frac{1.4693}{302.65}=0.004855 \text {. }
$$

Der Unterschied von etwas über $1 \%$ überschreitet nicht die Analysenfehler, woraus folgt, daß die molare Löslichkeit der untersuchten Nitrate bis zu dieser Genauigkeit als gleich erwiesen wurde.

Der Vergleich des Gewichtes der gesättigten Lösungen mit Hilfe eines Pyknometers läßt sich natürlich viel exakter durchführen, und wir wollen ähnlich aber genauer als im Kapitel 2 auf Grund unserer Analysen berechnen, wie groß der Unterschied im Gewicht der Lösungen für die betreffenden zwei Bleiarten in dem benutzten Pyknometer, dessen Inhalt $9.9950 \mathrm{ccm}$ betrug, sein müßte.

Nimmt man das Mittel der Resultate der obigen zwei Analysen, $1.4716 \mathrm{~g}$ Sulfat für $3.0445 \mathrm{ccm}$ der Nitratlösungen und rechnet mit dem mittleren Molekulargewicht 302.93, so ergibt sich, daß in $1 \mathrm{ccm}$ der gesättigten Nitratlösungen $\frac{1.4716}{302.93 \cdot 3.0445}=0.0015956$ Grammatome Blei enthalten sind. Da der Gewichtsunterschied der zwei Bleie pro Grammatom 207.15 - 206.59=0.56 $\mathrm{g}$ beträgt, erhält man für $9.9950 \mathrm{ccm}$ der Lösungen einen zu erwartenden Gewichtsunterschied von

$$
0.56 \cdot 0.00160 \cdot 10.0 \cdot 1000=9.0 \mathrm{mg} \text {. }
$$

Wir lassen nun die Resultate der Pyknometerversuche folgen.

\section{Gewöhnliches Bleinitrat.}

$$
\begin{aligned}
& \text { Präparat C. „,kalt“ ... } 22.7515 \mathrm{~g} \\
& \text {,kalt" . . . } \frac{22.7512 \mathrm{~g}}{\text { Mittel }} \\
& \text { Präparat D. „kalt" . . . } 22.7520 \mathrm{~g} \\
& \text {,,kalt" : . . . } 22.7530 \mathrm{~g} \\
& \text { „heiß"... . 22.7528 g } \\
& \text { Mittel } 22.7526 \mathrm{~g}
\end{aligned}
$$


Carnotitbleinitrat.

Präparat A.

$$
\begin{array}{r}
\text { „kalt" . . . . . } 22.7389 \mathrm{~g} \\
\text { "kalt" . . . . . } 22.7388 \mathrm{~g} \\
\text { „heiß" . . . . } 22.7376 \mathrm{~g} \\
\text { Mittel } 22.7384 \mathrm{~g}
\end{array}
$$

Präparat B.

$$
\begin{array}{r}
\text { "heiß" . . . . } 22.7448 \mathrm{~g} \\
\text { "halt" . . . . } 22.7462 \mathrm{~g} \\
\text { Mittel } 22.7455 \mathrm{~g}
\end{array}
$$

Wie ersichtlich, ist der Unterschied zwischen den Mittelwerten der auf völlig verschiedenem Wege erhaltenen Präparate $C$ und $D$ nicht um Vieles größer als die Versuchsfehler bei demselben Präparat. Vergleicht man nun das gemeinsame Mittel 22.7520 dieser Versuche mit dem Mittel der Resultate des Präparates A, 22.7384, so erhäıt man den Unterschied von $13.6 \mathrm{mg}$, während theoretisch $9 \mathrm{mg}$ zu erwarten waren.

Da ein zu niedriger Wert des Präparates A eventuell von kleinen Mengen Salpetersäure, die die Löslichkeit des Nitrates stark beeinflußt, herrühren konnte, wurde A noch zweimal kristallisiert, und das erhaltene Präparat B ergab in der Tat den wesentlich höheren Wert 22.7455. Jetzt war aber der Unterschied zwischen diesem Präparat und dem gewöhnlichen Nitrat nur $6.6 \mathrm{mg}$. Durch weitere Kristallisationen beider Präparate und eventuell durch Herstellung neuer unabhängiger Präparate sollten diese Schwankungen aufgeklärt werden. Wir mußten aber an dieser Stelle die Versuche unterbrechen. Sie wurden im Herbst 1914 von dem einen von uns und Herrn Dr. Lembert aufgenommen. Es wurde sowohl das Präparat $B$ als auch ein Gemisch von $C$ und $D$ je zweimal kristallisiert, wobei für die gesättigten Lösungen in dem obigen Pyknometer ein Gewichtsunterschied von $11.2 \mathrm{mg}$ resultierte.

Aus allen diesen Versuchen war ersichtlich, daß der gesuchte Unterschied der Dichte gesättigter Lösungen der Nitrate isotoper Bleiarten in der Tat existiert, und daß wir auch dessen Größenordnung richtig erhalten haben. Zu einer einigermaßen genauen Messung dieses Unterschiedes war aber die Reproduzierbarkeit unserer Versuche noch nicht genügend. Da die Schwankungen der Resultate bei ein und demselben Präparat beträchtlich kleiner als bei verschiedenen Präparaten waren, ergab 
296 K. Fajans u. J. Fischler. Über die Löslichkeit der Salze isotoper Elemente.

sich für die Fortsetzung der Arbeit die Forderung, besonders die präparative Seite zu beachten.

Was noch den Vergleich der molaren Löslichkeit der Nitrate der benutzten zwei Bleiarten anbelangt, so kann man aus den obigen Dichtebestimmungen folgern ${ }^{1}$, daß ein etwaiger Unterschied darin kleiner als $1 \%$ ist ${ }^{2}$

Herrn Dr. M. Lembe bei der Endreinigung der Präparate unseren besten Dank aussprechen.

1 Genauere Diskussion vgl. Kapitel VIII der folgenden Arbeit.

2 Dieses Resultat ist schon kurz mitgeteilt worden (Physik. Zeitschr. 15 (1914), 935).

Karlsruhe, Institut für physikalische Chemie der technischen Hochschule.

Bei der Redaktion eingegangen am 7. März 1916. 